\title{
Fasten: Reinigung für Körper und Seele
}

\author{
Starten Sie in eine gesündere und bewusstere \\ Lebensweise - Fasten ist ein guter Initialzünder
}

\begin{abstract}
Liebe Patientin, lieber Patient, haben auch Sie schon öfter darüber nachgedacht, sich gesünder zu ernähren, weniger Kaffee oder Alkohol zu trinken, weniger Süßigkeiten zu essen? Das Fasten bietet eine gute Möglichkeit, den Körper zu entlasten und neu in eine gesündere und bewusstere Lebensweise zu starten. Dabei kann Ihnen diese Patienteninformation eine Hilfestellung geben.
\end{abstract}

Es gibt verschiedene Formen des Fastens auch hinsichtlich der Fastendauer. Eine Fastenwoche wie im Beispiel unten gemäß dem Buchinger Heilfasten ist sehr gut für Anfänger geeignet.

Fasten ist für fast alle Menschen möglich. Trotzdem sollten Sie vor dem Fasten mit Ihrem Arzt sprechen, insbesondere wenn Erkrankungen bestehen. Dann sollte Ihre Fastenkur durch einen erfahrenen Fastentherapeuten begleitet werden.

\section{Fastenplan}

Fasten bedeutet keineswegs Hungern. Es ist lediglich der Verzicht auf feste Nahrung über 5 Tage, mit 1 Entlastungstag davor und 2 Aufbautagen im Anschluss. Während des Fastens sollten Sie möglichst nicht arbeiten und einige Grundregeln beachten, um Ihre persönliche Fastenzeit sicher, entspannt und erfolgreich zu gestalten.

\section{Entlastungstag}

Die Fastenwoche beginnt mit einem Entlastungstag, an dem zur Einstimmung nur noch leichte, frische Nahrung gegessen wird, die zu einer weichen Darmfüllung führt. An diesem Tag stehen das Ablösen vom Alltag, Ruhefinden und die Einstimmung auf die folgenden Fastentage im Mittelpunkt.
Ernährungsplan Entlastungstag

- morgens: Obst und Nüsse

- mittags: Rohkostplatte, Kartoffeln, Gemüse, Quarkspeise

- nachmittags: 1 Apfel, 10 Nüsse

- abends: Obst oder Obstsalat mit Leinsamen, 125 mg Joghurt, Knäckebrot

- Es ist wichtig, mindestens 31 zu trinken.

\section{Erster Fastentag}

Der erste Fastentag beginnt mit einer gründlichen Darmentleerung. Damit werden Gärungs- und Fäulnisprozesse durch im Körper verbleibenden Darminhalt vermieden, der Darm entlastet und das Hungergefühl reduziert.

Zum Abführen können Sie 40 g Glaubersalz in $750 \mathrm{ml}$ Wasser auflösen, einige Spritzer Zitronensaft zugeben und innerhalb einer Viertelstunde trinken. Vorher, zwischendurch und danach bietet sich bei Bedarf Pfefferminztee an, um den Salzgeschmack abzumildern. Innerhalb der nächsten 1-3 Stunden sollten nun mehrere durchfallartige Entleerungen erfolgen, die gelegentlich bis zum Nachmittag anhalten. Bei empfindlichem Darm reicht statt Glaubersalz evtl. bereits 1 Glas Sauerkrautsaft zum Abführen aus. Sie können aber auch einen Einlauf oder ein salinisches Klysma aus der Apotheke zum Abführen anwenden.

Trinken Sie mindestens 3 1, um den Flüssigkeitsverlust auszugleichen. Sollte es zu Bauchschmerzen kommen, hilft Bettruhe mit einer Wärmflasche auf dem Bauch. Achten Sie darauf, dass Ihre Füße warm sind, auch dazu bietet sich eine Wärmflasche an.

Heute nehmen Sie keine feste Nahrung mehr zu sich: Der Körper schaltet von Nahrungsaufnahme auf Ausscheidung um. Die Ernährung von innen beginnt, der Hunger

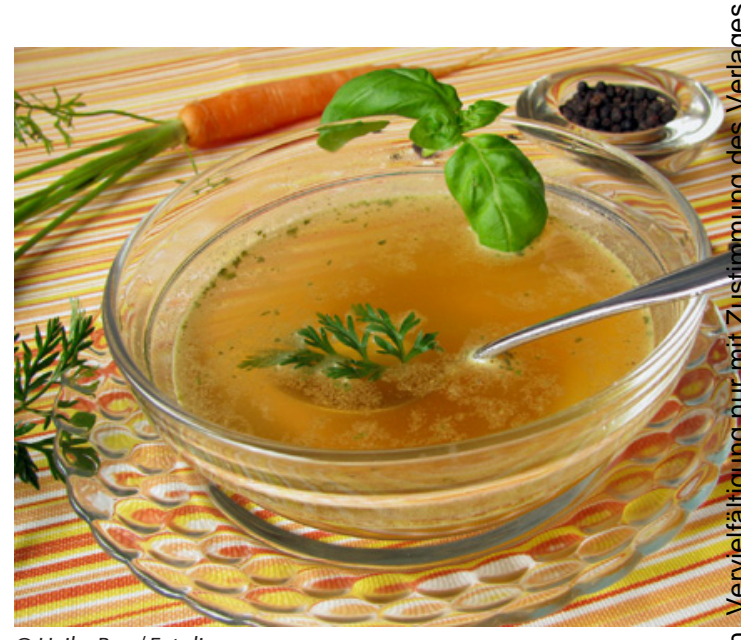

(c) Heike Rau/Fotolia.com

verschwindet schrittweise. Sie leben jetzt aus sich selbst. Gehen Sie spazieren, lesen oder ruhen Sie, wenn Ihnen danach zumute ist. Muten Sie sich bis zum Ende der Fastenwoche keine zu großen Anstrengungen oder starke Wärmereize (Vollbad, Sauna) mehr zu und gehen Sie früh zu Bett (die Leber entgiftet am besten im Liegen). Ernährung s. Ernährungsplan 1.-5. Fastentag.

\section{Zweiter Fastentag}

Heute liegt der Schwerpunkt auf der Durchspülung von Nieren und Geweben. Trinken Sie mindestens 3 1. Der Urin sollte eine helle Farbe haben, ansonsten mehr trinken. Sorgen Sie für warme Hände und Füße, ruhen Sie viel und unternehmen Sie Spaziergänge. Ernährung s. Ernährungsplan 1.-5. Fastentag.

\section{Dritter Fastentag}

Heute wird der Darm erneut entleert. Trinken Sie dazu morgens 1 Glas Molke, Sauerkraut- oder Pflaumensaft oder, falls dies nicht spontan abführend wirkt, Glaubersalz wie für den ersten Fastentag beschrieben. Sie können alternativ auch einen Einlauf oder ein Klysma anwenden. Trinken Sie mindestens 3 1, um den Flüssigkeitsverlust auszugleichen. Bewegen Sie sich viel an der frischen Luft und legen Sie eine Mittagsruhe ein, bürsten und ölen Sie Ihren Körper. 
Rezepte: Gemüsebrühen für

Fastentage

Kartoffelbrühe (4 Portionen)

- 1 I Wasser

- $250 \mathrm{~g}$ Kartoffeln

- 2 Karotten

- $1 / 2$ Stange Lauch

- 1 Petersilienwurzel

- $1 / 4$ Knolle Sellerie

- 1 TL Gemüsebrühe

- je $1 / 2$ TL Kümmel und Majoran

- Salz, Muskatnuss

- 2 TL Hefeflocken

- 4 TL frisch gehackte Petersilie

Gemüse gut waschen und klein schneiden, Wasser aufkochen, Gemüse zufügen und 10-20 Minuten gar kochen. Suppe vom Herd nehmen, durchseihen und mit Gewürzen abschmecken, Hefeflocken und Petersilie darüberstreuen.

Karottenbrühe (4 Portionen)
- 1 I Wasser
- $250 \mathrm{~g}$ Karotten
- $1 \frac{2}{2}$ Stange Lauch
- 1 Petersilienwurzel
- $100 \mathrm{~g}$ Sellerie
- 1 TL Gemüsebrühe
- Salz, Muskatnuss
$=2$ TL Hefeflocken
= 4 TL frisch gehackte Petersilie
Zubereitung wie Kartoffelbrühe.

Selleriebrühe (4 Portionen)

- 1 I Wasser

- $250 \mathrm{~g}$ Sellerieknolle

- etwas Lauch und Karotten

- je 1 TL Majoran und Kümmel

- 1 TL Gemüsebrühe

- 2 TL Hefeflocken

- 4 TL frisch gehackte Petersilie

Das Gemüse gut waschen, ungeschält klein schneiden. Wasser zum Kochen bringen, Gemüse zufügen und darin 10-20 Minuten gar kochen. Suppe vom Herd nehmen, durchseihen, Brühe mit Gewürzen hinzufügen, Hefeflocken und die Petersilie darüberstreuen.

Tomatenbrühe (4 Portionen)

- 1 I Wasser

- $500 \mathrm{~g}$ Tomaten

- 1 Knoblauchzehe

- je $100 \mathrm{~g}$ Lauch, Sellerie und Karotte

- Salz

- 1 TL Gemüsebrühe

- je 2 TL Oregano oder Majoran

- 2 TL Hefeflocken

Tomaten gut waschen, von Stielansätzen

befreien und würfeln, Knoblauchzehe klein hacken. Das restliche Gemüse gut waschen und ungeschält klein schneiden. Wasser zum Kochen bringen, Tomaten, Knoblauch und Gemüse zufügen und darin 10-20 Minuten gar kochen. Die Suppe vom Herd nehmen, durchseihen, mit Gewürzen abschmecken und Hefeflocken darüberstreuen.
Außerdem bieten sich Wechselduschen (Kalt-warm-Wechsel) an. Ernährung s. Ernährungsplan 1.-5. Fastentag.

\section{Vierter und fünfter Fastentag}

Auch an diesen Tagen gilt: Ausreichend trinken, bewegen und ruhen. Ernährung s. Ernährungsplan 1.-5. Fastentag. Am fünften Fastentag wird morgens erneut mit einem Glas Molke, Sauerkraut- oder Pflaumensaft abgeführt oder, falls dies nicht ausreicht, mit Glaubersalz (Durchführung s. erster Fastentag).

\section{Ernährungsplan 1.-5. Fastentag}

- morgens: 2 Tassen Kräutertee (z. B. Brennnessel, Kamille, Malve, Melisse oder Ginseng) oder milder schwarzer Tee mit Zitrone, erlaubt ist dazu je $1 / 2$ TL Honig

- zwischendurch: reichlich Wasser oder Mineralwasser ohne Kohlensäure, gelegentlich 1 Zitronenschnitz aussaugen

- mittags: 250 ml Gemüsesuppe, selbst zubereitet in verschiedenen Variationen ( $\mathrm{s}$. Kasten: Gemüsebrühen für Fastentage), oder $250 \mathrm{ml}$ Gemüsefrischsaft mit Wasser aufgefüllt

- nachmittags: 2 Tassen Früchtetee (Hagebutten, Fenchel oder Apfelschalen), Kräutertee oder milder Schwarztee (nicht nach 16 Uhr) mit Zitrone und je bis zu $1 \frac{1}{2}$ TL Honig

- abends: 250 ml Obstsaft nach Geschmack mit Mineralwasser verdünnt, nach Wahl heiß oder kalt, alternativ Gemüsebrühe oder -saft

Alle Fastengetränke schluckweise trinken. Jeden Schluck „kauen“, das heißt, im Mund vorwärmen oder kühlen. Genießen und langsam trinken.

\section{Erster Aufbautag}

Trinken Sie weiterhin reichlich. Bewegen Sie sich vor dem Fastenbrechen am Vormittag ausgiebig (Sport oder Spaziergang) und gönnen Sie sich auch eine Mittagsruhe. Kalte Güsse aktivieren zusätzlich Ihren Stoffwechsel. Essen Sie achtsam: Wenig ist viel.

Ernährungsplan 1. Aufbautag

- morgens: Morgentee

- vormittags: Fastenbrechen mit 1-2 gut gereiften Äpfeln

- mittags: pürierte Kartoffel-Gemüse-Suppe - nachmittags: trinken wie bisher

- abends: Tomatensuppe, Knäckebrot, eingeweichtes Trockenobst

\section{Zweiter Aufbautag}

Vermeiden Sie auch heute Anstrengungen. Nutzen Sie hingegen anregende Maßnahmen wie lockere Bewegung (z. B. Spaziergang) an der frischen Luft, kalte Güsse und Körperbürstungen. Ein Gewichtsanstieg ist normal, da sich der Darm wieder füllt. Nach dem zweiten Aufbautag nehmen Sie schrittweise wieder Ihre normale Ernährung auf.

\section{Ernährungsplan 2. Aufbautag}

- morgens: Morgengetränk, 1 Glas Molke oder Sauerkrautsaft oder 2 Scheiben Knäckebrot mit Kräuterquark

- vormittags: Wasser zwischendurch

- mittags: Salat, Kartoffeln, Gemüse, Naturjoghurt

- Nachmittags: Kräutertee

- abends: Rohkost, Gemüsesuppe, Dickmilch, Knäckebrot

Christiana Purol, HP

Wismarer Str. 44, 12207 Berlin

info@purol.de

www.purol.de

Online zu finden unter

http://dx.doi.org/10.1055/s-0034-1368132

Anmerkungen Ihres Arztes

Mit den besten Grüßen überreicht durch: 